\title{
Actualización en diagnóstico e intervención temprana del Trastorno del Espectro Autista
}

\section{Update in diagnosis and early intervention of Autistic Spectrum Disorder}

\author{
Valeria Rojas $^{\mathrm{a}}$, Andrea Rivera ${ }^{\mathrm{b}}$, Nelson Nilo ${ }^{\mathrm{c}}$ \\ aHospital Gustavo Fricke, Hospital Carlos Van Buren. Universidad de Valparaíso \\ bPsicóloga. Universidad Viña del Mar \\ 'Terapeuta Ocupacional. Hospital Gustavo Fricke
}

Recibido: 13 de junio de 2019; Aceptado: 8 de agosto de 2019

\section{Resumen}

El Trastorno del Espectro Autista (TEA) es una alteración del neurodesarollo que afecta las áreas de comunicación social y conducta, las cuales se manifiestan de manera heterogénea en cada niño y con una amplia gama de niveles de funcionalidad. En la última década se han hecho avances significativos en la detección temprana de señales de riesgo, favoreciendo la realización de diagnósticos precoz. Esto ha permitido el acceso a intervenciones que capitalizan la neuroplasticidad de esta etapa del desarrollo, planteando la posibilidad de mitigar la completa manifestación del trastorno. Los objetivos de esta actualización son revisar herramientas de diagnóstico precoz y modelos de intervención temprana, y analizar cómo implementar intervenciones basadas en la evidencia en un contexto sanitario de un país como Chile.
Palabras clave:

Autismo;

detección precoz;

intervención temprana; salud pública

\begin{abstract}
Autism Spectrum Disorder (ASD) is a neurodevelopmental condition that affects the social communication and behavior areas. Its symptomatology display heterogeneity and a wide range of functionality levels in each child. In the last decade, significant advances have been made in the early detection of risk signs, favoring early diagnosis. This has allowed access to interventions that capitalize neuroplasticity of this stage of development, raising the possibility of mitigating the full manifestation of the disorder. The objective of this update is to review early diagnostic tools and early intervention models and to analyze how to implement evidence-based interventions in a health context in a country like Chile.
\end{abstract}

Keywords: Autism; early intervention; early detection; public health 


\section{Introducción}

El Trastorno del Espectro Autista (TEA) es una alteración en la trayectoria del neurodesarrollo que tiene manifestaciones observables en la conducta desde edades tempranas, determinando interferencias clínicamente significativas en la interacción y comunicación social, además de la presencia de comportamientos e intereses anormales y repetitivos ${ }^{1}$.

En ocasiones este diagnóstico puede cursar con discapacidad intelectual, dificultades en el lenguaje, trastornos psiquiátricos y de comportamiento, alteraciones en el sueño y alimentación, las que pueden estar presentes hasta en el 70\% de los casos ${ }^{2}$. Al ser un trastorno de carácter crónico, los pacientes van a requerir apoyos tanto familiares como comunitarios, estimándose un gasto anual de $\$ 11,5$ - 60,9 billones de dólares por persona ${ }^{3}$.

En países como Estados Unidos, se ha observado un alza en la prevalencia del diagnóstico, desde las primeras mediciones realizadas en el año 2000, con un 30.8 por $10,000^{4}$, hasta la última medición realizada el 2018 por el $\mathrm{CDC}^{3}$, el cual calculó una prevalencia de $1 / 59$ escolares de 8 años.

Desde la primera aparición del término autismo, de la mano de Leo Kanner en 1943, nuestra comprensión de este fenómeno ha evolucionado. Lo que en un principio era considerado como una forma de esquizofrenia infantil resultado de un estilo parental frío, ahora es considerado como un trastorno del neurodesarrollo, con un amplio espectro de niveles de funcionamiento.

La evolución del conocimiento del TEA se ha visto reflejada en los avances respecto a las estrategias para favorecer aprendizajes tempranos, mejorar la adaptabilidad y aumentar la calidad de vida de los niños con TEA. Realizar algún tipo de intervención especializada podría tener importantes repercusiones, y en alrededor del $25 \%$ de los casos podría significar la pérdida de los criterios diagnósticos de TEA en el transcurso de la vida ${ }^{5}$.

Uno de los avances más interesantes es la creciente evidencia respecto a cómo el TEA puede ser diagnosticado de manera confiable a partir de los $14 \operatorname{meses}^{6} \mathrm{y}$ los beneficios sustanciales que han reportado estudios de intervención temprana realizados con niños incluso menores de 15 meses $^{7}$. Estos estudios demuestran que gracias a la neuroplasticidad, intervenciones realizadas antes de los 2 o 3 años podrían contrarrestar la cascada de alteraciones en el desarrollo, propias del TEA, mitigando la completa manifestación de la sintomatología ${ }^{8}$.

El siguiente manuscrito tiene por objetivos revisar las herramientas de diagnóstico precoz y modelos de intervención temprana, y analizar cómo implementar las intervenciones basadas en la evidencia en un contexto sanitario de un país como Chile.

\section{Diagnóstico del Trastorno del Espectro Autista}

El TEA constituye una amplia gama de manifestaciones clínicas a nivel de comunicación, interacción social y comportamiento, las cuales se expresan de manera heterogénea dependiendo del individuo y de la etapa del ciclo vital ${ }^{1}$. Actualmente no existe una prueba médica que nos permita identificar biomarcadores de TEA. Muchas veces, especialmente en niños mayores, la anamnesis que recoge la historia del neurodesarrollo y pesquisa alteraciones en comunicación, habilidades sociales y comportamientos restrictivos, será suficiente para hacer el diagnóstico de TEA. Cuando son más pequeños o existen dudas diagnósticas, idealmente el diagnóstico debe ser hecho por un equipo multidisciplinario que incluye neurólogos y/o psiquiatras infanto-juveniles, psicólogos, fonoaudiólogos y terapeutas ocupacionales, en base a entrevistas y observaciones estructuradas como la entrevista de diagnóstico de autismo ADI- $\mathrm{R}^{9}$ y el Test de ADOS- $2^{10}$. Este último consiste en un protocolo de observación de la comunicación y habilidades sociales. El uso estandarizado de estas pruebas (como Modulo T-1-2 del ADOS-2) requiere que los evaluados puedan caminar de manera independiente, y que no tengan deficiencias auditivas ni visuales. A su vez, la prueba debe ser aplicada por personal altamente capacitado y muy experimentado ${ }^{11}$.

Las pruebas descritas anteriormente suelen ser las más definitorias a la hora de realizar un diagnóstico confiable, e incluso tienen la facultad de identificar el riesgo de TEA a partir de los 12 meses $^{10}$. Sin embargo, estudios realizados en Estados Unidos ${ }^{12}$, o el Reino Unido $^{13,14}$ siguen evidenciando demoras en el proceso de diagnóstico, especialmente si los niños no tienen dificultades cognitivas y presentan lenguaje expresivo verbal ${ }^{13}$. En el caso de Estados Unidos, la edad promedio en la cual los niños reciben el diagnóstico es a los 4,5 años ${ }^{12}$ y en el caso del Reino Unido es de 4.3 años $^{13}$. Se ha descrito que desde que los padres se acercan por primera vez a un profesional de la salud con preocupaciones relacionadas al TEA, hasta la confirmación del diagnóstico existe un promedio de 3,5 años ${ }^{13}$. Esto es especialmente grave si pensamos que existe evidencia robusta que nos indica que mientras antes comiencen las intervenciones, aumenta la posibilidad de disminuir la severidad de la sintomatología y evitar completa manifestación del trastorno ${ }^{7,8}$. A su vez, la intervención temprana supone un enfoque más eficiente en términos de costo y beneficio al reducir la cantidad de intervenciones necesarias a futuro ${ }^{5}$. 
Gracias a la creciente evidencia sobre la aparición de signos de TEA al final del primer año de vida, como alteraciones en el comportamiento, déficit en la comunicación social ${ }^{15}$, movimiento corporal inusual, regulación emocional atípica y reducido control motor ${ }^{16}$, nuestra comprensión de cómo emergen y evolucionan los síntomas de TEA en edades tempranas ha aumentado.

En este escenario, y en coherencia con las recomendaciones de la Academia Americana de Pediatría $(\mathrm{AAP})^{17}$, la cual orienta a realizar pruebas de screening a los 18 y 24 meses, es que la investigación científica ha orientado sus esfuerzos a crear evaluaciones tempranas que sean precisas y clínicamente funcionales en contextos de salud comunitarios.

Entre los test de screening más usado en la población general y validado recientemente en Chile $^{18}$, se encuentra el M-CHAT-R/ ${ }^{19}$. Este cuestionario está dirigido a padres o cuidadores de niños de entre 16 y 30 meses y ha mostrado un alto poder predictivo cuando se aplicaba en conjunto con evaluaciones rutinarias del desarrollo ${ }^{19}$.

Otro campo de investigación que se encuentra en desarrollo es la búsqueda de biomarcadores que faciliten el proceso diagnóstico. Debido a las anormalidades neuroanatómicas observadas de manera consistente a lo largo del desarrollo y la presencia de mecanismos biológicos, se espera que estas características pudieran proveer de indicios o marcas medibles incluso antes de que los marcadores comportamentales del trastorno aparezcan, favoreciendo el diagnóstico temprano ${ }^{20}$. Dentro de este campo de investigación, la técnica del registro de los movimientos oculares (eye-tracking, ET) ha demostrado potencial para identificar y caracterizar el TEA a un nivel temprano ${ }^{21-22}$. Esta herramienta está diseñada para detectar patrones visuales anormales asociados con el trastorno, en donde la fijación ocular de los pacientes con TEA está orientada en mayor medida hacia los estímulos no sociales en comparación con los sociales. El ET es una técnica no invasiva, que no requiere respuestas motoras avanzadas ni dominio del lenguaje ${ }^{23}$, por lo que los avances en la masificación de estas tecnologías podrían llegar a tener beneficios importantes a futuro en el contexto de salud pública.

El estudio de marcadores biológicos y comportamentales tempranos que permitan identificar el trastorno antes de los 12 meses, de manera precisa y clínicamente funcionales en contextos de salud comunitarios, continúan siendo campos de investigación en desarrollo. Sin embargo, es necesario pensar que las estrategias de detección temprana no se reducen a la elección de una herramienta, si no que deben basarse en acciones coordinadas y ser planificadas e implementadas en los dispositivos de salud de manera general. Esto implica una serie de acciones en diferentes niveles, como capacitación del personal, claridad en los conductos de derivación y la creación de equipos especializados.

\section{Intervención Temprana}

Con el aumento de la prevalencia y la creciente evidencia que el TEA puede ser diagnosticado de manera estable a los 2 años ${ }^{10}$, la comunidad científica ha debido enfocar sus esfuerzos en el diseño y evaluación de intervenciones pensadas especialmente para estas etapas del ciclo vital.

En cuanto al universo de intervenciones diseñadas para edades tempranas, una de las distinciones que se puede realizar tiene que ver con las estrategias que cada intervención utiliza para conseguir los aprendizajes deseados, en donde se pueden diferenciar dos grandes categorías: las intervenciones conductuales tempranas e intensivas (Early Intensive Behavioral Intervention, EIBI $)^{24}$, y las intervenciones del comportamiento basadas en el desarrollo ${ }^{25}$.

El enfoque EIBI se basa principalmente en los principios de modificación de la conducta o el análisis conductual aplicado (Applied Behavior Analysis, ABA), utilizando técnicas estructuradas como el refuerzo positivo, el modelamiento e incitación física. Entre los programas que utilizan esta filosofía, podemos nombrar al modelo de Lovaas ${ }^{26}$ y el entrenamiento de res-

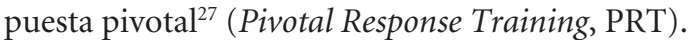

El modelo de Lovaas basa sus enseñanzas en el aprendizaje conductual sistemático a través de estrategias de enseñanza estructuradas (ensayo discreto y aprendizaje incidental), con una frecuencia intensiva, entre 20 y 40 horas a la semana, aplicable en diferentes contextos $^{26}$. Por otro lado el PRT, a pesar de basar sus fundamentos en el enfoque ABA, introduce elementos novedosos como la importancia de la motivación, y la capacidad de respuesta a múltiples claves ambientales. Este modelo propone trabajar en áreas pivotales del desarrollo, más que en habilidades específicas, con el objetivo de lograr mejoras colaterales en habilidades sociales, comunicativas y conductuales ${ }^{27}$.

Por otro lado, los programas basados en el desarrollo se caracterizan por ser poco estructurados. La enseñanza se realiza en entornos naturales y el control de la sesión es compartido con el niño, siendo su prioridad la comunicación social y la interacción ${ }^{25}$. Entre estos modelos encontramos la terapia de juego en el suelo $(\mathrm{DIR} / \mathrm{Floor} \text { time })^{28}$. Esta terapia basa sus principios en el desarrollo y en las relaciones. No plantea objetivos en áreas específicas del desarrollo, sino que destaca la importancia de acercar al niño a un mundo emocional compartido, favoreciendo el desarrollo y los intereses emocionales. 
También existen intervenciones que se encuentran entre estos dos polos. Estas estrategias añaden a las técnicas conductuales una visión comprensiva del desarrollo, en donde se considera la singularidad de los procesos cognitivos, comunicativos y de aprendizaje propias de los bebes e infantes ${ }^{29}$. Entre estos modelos "mixtos" de intervención, se encuentran el modelo SCERTS ${ }^{30}$ (Social Communication Emotional Regulation Transactional Support), el método TEACCH ${ }^{31} y$ el Early Start Denver Model (ESDM) $)^{32}$. El modelo SCERTS busca potenciar el desarrollo de las habilidades socioemocionales y comunicativas de las personas con TEA, a través de apoyos transaccionales ${ }^{30}$. Por otro lado, el método TEACCH se basa en aprendizaje estructurado el cual se realiza utilizando el perfil de aprendizaje propio del TEA, en donde se consideran sus fortalezas (procesamiento visual) y sus desafíos (comunicación social, atención y función ejecutiva). Se utiliza principalmente en ambientes educativos en donde los profesores pueden implementar estrategias como el uso de paneles de organización externa y apoyos visuales o escritos para complementar la orden verbal $^{31}$. En cuanto al ESDM, se trata de un modelo de intervención comprensiva diseñado específicamente para niños pequeños con TEA (12-48 meses), cuyo objetivo es acelerar el ritmo de desarrollo y "rellenar", o compensar las afectaciones producidas por la falta de motivación social que caracteriza al diagnóstico. Este modelo se nutre de los principios ABA, al planificar oportunidades de aprendizaje estructuradas y al contemplar elementos como las mediciones y evaluaciones de forma periódica. A su vez, prioriza la promoción de la comunicación social y la interacción en contextos naturales y adecuados al nivel de desarrollo del niño, en donde el control de la sesión es compartido y se fomenta el aspecto lúdico y placentero de estas oportunidades de aprendizaje. Este modelo capitaliza la neuroplasticidad de estas etapas, al sumergir al niño en interacciones sociales significativas y promotoras de aprendizajes $^{32}$.

Respecto a la efectividad de estos diferentes modelos de intervención, aquellos basados en la metodología ABA cuentan con evidencia suficiente como para ser recomendados ${ }^{33}$. Sin embargo, expertos advierten que estos formatos estructurados y altamente directivos podrían generar dependencia a claves específicas del entorno para responder y falta de espontaneidad e iniciativa en la comunicación ${ }^{34}$. Por otro lado, modelos combinados como el ESDM han sido evaluados favorablemente ${ }^{35}$, siendo catalogada como posiblemente eficaz ${ }^{33}$, o como potencialmente prometedora ${ }^{36}$, coincidiendo estas últimas publicaciones en la falta de mayor evidencia, especialmente ensayos de control aleatorio. Otros modelos con menores puntuaciones, pero aún así valorables son el método TEACCH, PRT y SCERTS ${ }^{35}$. Por otro lado, aquellas que presentan evidencia limitada y poco concluyente a la hora de reducir la sintomatología TEA, son las intervenciones sensoriales que provienen del campo de la terapia ocupacio$\mathrm{nal}^{37}$. Sin embargo, existen autores que las consideran como positivas dentro de un programa más amplio de trabajo, aunque no deben nunca suplir las intervenciones mencionadas anteriormente ${ }^{34}$.

Independiente del modelo a elegir por la familia o profesionales, existen recomendaciones generales de buena práctica para las intervenciones realizadas en esta población. Entre las más nombradas por la literatura está la necesidad de comenzar lo antes posible, incluso en el caso de aquellos niños que muestren sintomatología sub-clínica de TEA. El objetivo principal es que los niños y sus familias tengan acceso a intervenciones que permitan maximizar las experiencias de aprendizaje en el contexto de interacción social, permitiendo acelerar el proceso de desarrollo ${ }^{24,38,39}$.

El trabajo con padres ha sido objeto de varios estu$\operatorname{dios}^{40-44}$, mostrando un importante potencial al poder maximizar las oportunidades de aprendizaje, con intervenciones de baja intensidad. Estos resultados son positivos incluso cuando el entrenamiento para padres se realiza a través de una plataforma digital ${ }^{43}$, transformándose en una alternativa en contextos en donde el recurso profesional y económico es limitado. Dentro del trabajo con padres, existen recomendaciones específicas de buenas prácticas entre las cuales está que el entrenamiento de los padres sea práctico y basado en su propia interacción con el niño ${ }^{45} \mathrm{y}$ que los objetivos de trabajo sean acotados de manera de asegurar la consolidación de las habilidades de los niños ${ }^{5}$.

Otra recomendación que los expertos realizan es que las intervenciones deben contemplar objetivos en diversas áreas del desarrollo, enfocándose tanto en las características principales del TEA, como en la sintomatología asociada ${ }^{20,33}$. Es importante destacar que, independiente del modelo de intervención que se utilice, éste debe ser basado en la evidencia, además de contemplar un enfoque centrado en las necesidades de la familia y que incorpore un plan de intervención individualizado ${ }^{39}$.

Las recomendaciones descritas anteriormente son fruto de un avance considerable en el número de investigaciones que han destinado sus esfuerzos a evaluar de manera empírica los resultados de las intervenciones para niños con TEA. En la actualidad siguen existiendo preguntas sin respuesta que orientan las investigaciones futuras. Una de éstas es la necesidad de identificar los "ingredientes activos" de cada modelo de intervención en contraste con un grupo de control bien definido $^{33}$. Esto permitiría depurar y simplificar las intervenciones utilizando solo aquellos elementos que sî funcionan. Otra pregunta de investigación se relaciona 
con la necesidad de identificar de manera individual, en base a las características del niño y de la familia, la intervención de la cual más se beneficiarían ${ }^{16}$. Esto es relevante de cara a la heterogeneidad con la cual se manifiesta el trastorno considerando el fenotipo y las comorbilidades, además de rescatar la individualidad de cada sistema familiar y sus preferencias.

Para finalizar, podemos plantear que el gran reto de las investigaciones es el estudio de cómo modelos de intervención basados en la evidenciase pueden implementar en contextos como Salud y Educación, asegurando una entrega más masiva. Esto es sumamente relevante considerando la acotada ventana de tiempo que tenemos para realizar intervenciones tempranas que puedan tener un impacto en la población afectada con el diagnóstico.

\section{Implementación en Chile}

Podemos decir que la investigación en TEA sigue un continuo en donde la producción del conocimiento tradicional, va desde la ciencia básica hasta el desarrollo de la intervención, luego a la investigación de la eficacia, y finalmente a los intentos de diseminación e implementación ${ }^{46}$.

A pesar de que ya existen estudios que se encuentran en esta última etapa en la producción de conocimientos, los cuales están orientados a evaluar la aceptabilidad y viabilidad de la implementación de modelos de intervención como el ESDM en contextos como Sa$\operatorname{lud}^{47,48}$ y Educación ${ }^{49,50}$, la mayoría de esta producción científica ha sido realizada en países desarrollados.

Implementar intervenciones con altos niveles de rigor en países de mediano y bajo ingreso es sumamente difícil debido a la falta de recursos ${ }^{51}$. A pesar de las dificultades, existen estudios como el realizado en 2015 por Divan ${ }^{52}$ en India, cuyo objetivo es implementar una adaptación de un modelo basado en la evidencia, en un contexto de bajos recursos. La viabilidad de la intervención está basada en dos aspectos claves, que la intervención fuera implementada por los padres, y que ellos fueran capacitados por personal no especialista. Esta experiencia ilustra cómo es posible adaptar modelos de intervención basados en la evidencia, permitiendo mayor accesibilidad y factibilidad en contextos de bajos recursos.

En Chile existe hasta la fecha sólo una experiencia en la implementación de una adaptación de un modelo basado en la evidencia en contexto de salud pública. Esta iniciativa está siendo realizada por el Servicio de Salud Viña del Mar Quillota (SSVQ) desde el año 2017.

Durante el año 2017-2018 se han ingresado 50 niños, pertenecientes a las comunas de Quillota y Quilpué, con promedio de edad de 30 meses, quienes reci- bieron intervención temprana en las Salas de Estimulación Del Chile Crece Contigo, en un modelo adaptado del ESDM. Las terapias fueron realizadas con una duración semanal de 16-20 sesiones, por profesionales educadoras de párvulos, o terapeutas ocupacionales, o fonoaudiólogas, dependiendo de la realidad de cada comuna, con la participación activa de los padres. Estas profesionales fueron previamente capacitadas por el equipo TEA del Hospital Gustavo Fricke y cada sesión fue videada, para luego ser supervisada en forma on-line por profesional experto del nivel secundario. De los 50 niños ingresados, a 23 de ellos se les realizo Test ADOS-2 al inicio y al final de las terapias, disminuyendo en 22/23 niños la severidad de los síntomas marcadores de autismo.

Similar a lo reportado por Divan ${ }^{52}$, el programa del SSVQ descrito anteriormente debió realizar ciertas adaptaciones que permitieran implementar intervenciones basadas en la evidencia, en contextos con pocos recursos. La primera fue ajustar un modelo de tratamiento compresivo como el ESDM a los requerimientos del contexto de salud primaria, específicamente respecto a la intensidad de las intervenciones. En su versión original el ESDM propone una intensidad de 20 horas a la semana ${ }^{32}$, lo cual es inviable en contexto de salud pública. Para dar solución a este problema se optó por basar la intervención en el entrenamiento de padres, el cual ha mostrado ser exitosos a la hora de generar cambios con intervenciones de baja frecuencia e intensidad ${ }^{40-44}$. La segunda adaptación fue entrenar a profesionales de la salud no especialistas en TEA, utilizando las lógicas de cambio de tareas (Task-Shiftting). Esta es una recomendación realizada por la Organización Mundial de la Salud ${ }^{53}$, cuyo objetivo es optimizar la entrega de servicios de salud a la comunidad, en contextos en donde existe escasez de especialistas. Este proceso consiste en la redistribución de tareas entre el personal de la salud, en donde tareas específicas que son realizadas por personal altamente entrenado, se reasignan a personal con menos especialidad los cuales pasan por un proceso de formación breve e intensiva.

Las estrategias para optimizar recursos utilizadas por Divan ${ }^{52}$ y por el plan piloto descrito anteriormente, han mostrado ser pertinentes en el contexto actual chileno el cual está marcado por la precariedad y la escasa investigación. Muestra de esto, es que en nuestro país aún no existen antecedentes respecto de la prevalencia de TEA, siendo la referencia más aproximada, la Guía de Práctica Clínica de Detección y Diagnóstico Oportuno de los Trastornos de Espectro Autista ${ }^{54}$, publicada el año 2011 por el Ministerio de Salud. En ésta se estimó una prevalencia el año 2007 de 89,6 casos por cada 10.000 recién nacidos vivo. Esta es una cifra extrapolada basada en prevalencias obtenidas de estudios internacionales. Sumado a esto, podemos decir que al 
no considerarse una meta sanitaria, se observa un déficit en el desarrollo, a nivel público, de políticas que favorezcan la estimulación temprana e intervención y apoyo para sujetos con dicho diagnóstico.

Esta Actualidad representa un esfuerzo por difundir el conocimiento que se ha producido en los últimos años en torno a los hitos más importantes en diagnóstico e intervenciones tempranas. En este contexto se plantea el desafío de democratizar el acceso a intervenciones tempranas y de calidad para los niños con TEA en nuestro país, especialmente respecto a la adecuación de modelos de intervención basados en la evidencia, con buenos niveles de control y rigurosidad, a contextos de salud pública, considerando los recursos limitados.

\section{Conflicto de intereses}

Los autores declaran no tener conflicto de intereses.

\section{Referencias}

1. American Psychiatric Association. Diagnostic and statistical manual of mental disorders (5th ed.). Arlington, VA: American Psychiatric Association. 2013.

2. Kendall T, Megnin-Viggars O, Gould N, Taylor C, Burt L, Baird G. Management of autism in children and young people: summary of NICE and SCIE guidance. BMJ, 2013. 347(aug28 2), f4865-f4865. http://dx.doi.org/10.1136/bmj.f4865.

3. Data and Statistics on Autism Spectrum Disorder | CDC [Internet]. Centers for Disease Control and Prevention. 2019 [citado el 13 de mayo de 2019]. Disponible en: https://www.cdc.gov/ ncbddd/autism/data.html.

4. Baron-Cohen S, Wheelwright S, Cox A, et al. Early identification of autism by the Checklist for Autism in Toddlers (CHAT). J Royal Soc Med. 2000; 93(10):521-5.

5. Koegel L, Koegel R, Ashbaugh K, Bradshaw J. The importance of early identification and intervention for children with or at risk for autism spectrum disorders.Int J Speech Lang Pathol. 2013;16(1):50-6. http://dx.doi.org/ 10.3109/17549507.2013.861511.

6. Pierce K, Gazestani V, Bacon E, et al. Evaluation of the Diagnostic Stability of the Early Autism Spectrum Disorder Phenotype in the General Population Starting at 12 Months. JAMA Pediatr. 2019. doi: 10.1001/ jamapediatrics.2019.0624.

7. Rogers S, Vismara L, Wagner A, McCormick C, Young G, Ozonoff S. Autism Treatment in the First Year of Life: A Pilot Study of Infant Start, a Parent-Implemented Intervention for Symptomatic Infants. Rev J Autism Dev Disord. 2014;44(12):2981-95. http:// dx.doi.org/10.1007/s10803-014-2202-y.

8. Dawson G. Early behavioral intervention, brain plasticity, and the prevention of autism spectrum disorder.Dev Psychopathol. 2008;20(3):775-803.

9. Lord C, Rutter M, Le Couteur A. Autism Diagnostic Interview-Revised: A revised version of a diagnostic interview for caregivers of individuals with possible pervasive developmental disorders. Rev J Autism Dev Disord. 1994;24(5):659-85.

10. Lord C, Risi S, Lambrecht L, et al. The autism diagnostic observation schedulegeneric: A standard measure of social and communication deficits associated with the spectrum of autism. Rev J Autism DevDisord. 2000;30(3):205-23.

11. Kamp-Becker I, Albertowski K, Becker J, et al. Diagnostic accuracy of the ADOS and ADOS-2 in clinical practice. Eur Child Adolesc Psychiatry. 2018;27(9):1193-207.

12. Wingate M, Kirby R, Pettygrove S, et al. Developmental Disabilities Monitoring Network Surveillance Year 2010 Principal Investigators. Prevalence of autism spectrum disorder among children aged 8 years autism and developmental disabilities monitoring net- work, 11 sites, united states. MMWR CDC SurveillSumm. 2010;63(2):1-21.

13. Crane L, Chester J, Goddard L, Henry L, Hill E. Experiences of autism diagnosis: A survey of over 1000 parents in the United Kingdom. Autism. 2015;20(2):153-62.

14. Howlin, P., Moore, ADiagnosis in autism: a survey of over 1200 patients in the UK. Autism. 1997;1(2):135-62.

15. Saint-Georges C, Cassel RS, Cohen $\mathrm{D}$, et al. What studies of family home movies can teach us about autistic infants: a literature review.Res Autism SpectrDisord. 2010;4(3):355-66. doi: 10.1016/j.rasd.2009.10.017.

16. Zwaigenbaum L, Bauman M, Choueiri R, et al. Early Identification and Interventions for Autism Spectrum Disorder: Executive Summary. Pediatrics. 2015;136(Supplement):S1-S9. doi: 10.1542/peds.2014-3667b.

17. Johnson CP, Myers SM; American Academy of Pediatrics Council on Children With Disabilities. Identification and evaluation of children with autism spectrum disorders. Pediatrics. 2007;120(5):1183-215.

18. Coelho-Medeiros M, Bronstein J, Aedo K, et al. Validación del M-CHAT-R/F como instrumento de tamizaje para detección precoz en niños con trastorno del espectro autista. Rev Chil de Pediatr 2018;90(5). doi:http://dx.doi.org/10.32641/rchped. v90i5.703.

19. Robins DL, Casagrande K, Barton M, Chen C-MA, Dumont-Mathieu T, Fein D. Validation of the modified checklist for autism in toddlers, revised with follow-up (M-CHAT-R/F).Pediatrics. 2014;133(1):37-45. doi: 10.1542/ peds.2013-1813.

20. Zwaigenbaum L, Bauman M, Fein D, et al. Early Screening of Autism Spectrum Disorder: Recommendations for Practice and Research. Pediatrics. 2015; 136(Supplement), S41-S59.doi: 10.1542/ peds. $2014-3667 \mathrm{~d}$.

21. Pierce K, Marinero S, Hazin R, McKenna, B, Barnes CC, Malige A. Eye tracking reveals abnormal visual preference for geometric images as an early biomarker of an autism spectrum disorder subtype associated with increased symptom severity.Biol Psychiatry 2016;79(8):65766. https://doi.org/10.1016/j. biopsych.2015.03.032.

22. Frazier TW, Klingemier EW, Beukemann $\mathrm{M}$, et al. Development of an Objective Autism Risk Index Using Remote Eye Tracking. J Am Acad Child Adolesc Psychiatry. 2016;55(4):3019. https://doi. org/10.1016/j.jaac.2016.01.011.

23. Moriuchi JM, Klin A, Jones W. Mechanisms of Diminished Attention to Eyes in Autism.Am J Psychiatry. 2017;174(1):26-35.

24. Reichow B, Barton E, Boyd B. Hume K. Early intensive behavioral intervention (EIBI) for young children with autism spectrum disorders (ASD): A Systematic Review. Campbell Systematic Reviews. 2014:9. DOI: 10.4073/csr.2014.9.

25. Ingersoll B, Dvortcsak A, Whalen C, Sikora D. The effects of a developmental, social-pragmatic language intervention on rate of expressive language production in young children with autistic spectrum disorders. Focus Autism Other Dev 
Disabl. 2005;20:213-22. doi:10.1177/1088 3576050200040301.

26. Smith T. Early and intensive behavioral intervention in autism. In J. R. Weisz \& A. E. Kazdin (Eds.), Evidence-based psychotherapies for children and adolescents (2nd ed., pp. 312-26). New York, NY: Guilford; 2010.

27. Koegel RL, Koegel LK. Pivotal response treatments for autism: Communication, social, and academic development. Baltimore, MD: Brookes; 2006.

28. Stanley Greenspan [Internet]. Stanley Greenspan. 2019 [citado el 14 junio de 2019]. Disponible en: https://www. stanleygreenspan.com/

29. Schreibman L, Dawson G, Stahmer A, et al. Naturalistic Developmental Behavioral Interventions: Empirically Validated Treatments for Autism Spectrum Disorder. J Autism Dev Disord. 2015; 45(8):2411-28. http://dx.doi.org/10.1007/ s10803-015-2407-8.

30. Prizant BM, Wetherby AM, Rubin E, Laurent A. The SCERTS model: A transactional, family-centered approach to enhancing communication and socioemotional abilities of children with autism spectrum disorder. Infants \& Young Children. 2003;16:296-316.

31. TEACCH $\mid$ Autism Speaks [Internet]. Autism Speaks. 2019 [citado el 7 Julio 2019]. Disponible en: https://www. autismspeaks.org/teacch-0.

32. Rogers S, Dawson G. Early Start Denver Model for young children with autism. New York: Guilford Press; 2010.

33. Smith, T., Ladarola, S. Evidence Base Update for Autism Spectrum Disorder. J Clin Child Psychol. 2015;44(6):897-922.

34. Pérez Á, Franco García R, García González V, et al. Manual TEA. $1^{\text {a }}$ Edición. Sevilla: Autismo Andalucía; 2018.

35. American Evaluation Association. Guiding principles for evaluators. Am Eval. 2008;29:233-4.

36. Waddington $\mathrm{H}$, van der Meer L, Sigafoos J. Effectiveness of the Early Start Denver Model: a Systematic Review. Rev J Autism Dev Disord. 2016;3(2):93-106. Retrieved from http://link.springer.com/10.1007/ s40489-016-0095-8.

37. Zimmer M, Desch L, Rosen LD, et al.
Sensory integration therapies for children with developmental and behavioral disorders. Pediatrics. 2012; 129(6):1186-9.

38. Warren Z, McPheeters M, Sathe N, FossFeig J, Glasser A, Veenstra-VanderWeele J. A Systematic Review of Early Intensive Intervention for Autism Spectrum Disorders. Pediatrics. 2011; 127(5):e130311.doi: 10.1542/peds.2011-0426.

39. National Research Council. Educating children with autism. Washington, US: National Academy Press; 2001.

40. Estes A, Vismara L, Mercado C, et al. The Impact of Parent-Delivered Intervention on Parents of Very Young Children with Autism. J Autism Dev Disord. 2013;44(2):353-65. doi: 10.1007/s10803013-1874-z

41. Rogers SJ, Estes A, Lord C, et al. Effects of a brief Early Start Denver Model (ESDM)-based parenting intervention on toddlers at risk for autism spectrum disorders: A randomized controlled trial.J Am Acad Child Adolesc Psychiatry. 2012;51(10):1052-65. https://doi. org/10.1016/j.jaac.2012.08.003.

42. Vismara L, Colombi C, Rogers S. Can one hour per week of therapy lead to lasting changes in young children with autism? Autism. 2009;13(1):93-115. doi: 10.1177/1362361307098516.

43. Vismara L, McCormick C, Wagner A, Monlux K, Nadhan A Young G. Telehealth Parent Training in the Early Start Denver Model: Results from a Randomized Controlled Study. Focus Autism Other Dev Disabl. 2016;33(2):6779. doi: $10.1177 / 1088357616651064$.

44. Zhou B, Xu Q, Li H, et al. Effects of Parent-Implemented Early Start Denver Model Intervention on Chinese Toddlers with Autism Spectrum Disorder: A NonRandomized Controlled Trial. Autism Res. 2018;11(4):654-66. doi: 10.1002/ aur.1917.

45. Kasari C, Gulsrud A, Paparella T, Hellemann G, Berry K. Randomized comparative efficacy study of parentmediated interventions for toddlers with autism.J Consult Clin Psychol. 2015;83(3):554-63. doi: 10.1037/ a0039080.

46. Landsverk J, Brown CH, Reutz JR,
Palinkas J, Horwitz SM. Design elements in implementation research: a structured review of child welfare and child mental health studies.Adm Policy Ment Health. 2011;38(1):54-63.

47. Colombi C, Narzisi A, Ruta L, et al. Implementation of the Early Start Denver Model in an Italian community. Autism. 2016;22(2):126-133. doi: $10.1177 / 1362361316665792$

48. Devescovi R, Monasta L, Mancini A, et al. Early diagnosis and Early Start Denver Model intervention in autism spectrum disorders delivered in an Italian Public Health System service.Neuropsychiatr Dis Treat. 2016;14(12):1379-84. doi: 10.2147/ ndt.s106850.

49. Vivanti G, Dissanayake C, Zierhut C, Rogers S. Brief Report: Predictors of Outcomes in the Early Start Denver Model Delivered in a Group Setting.J Autism Dev Disord. 2012;43(7):1717 1724.doi: 10.1007/s10803-012-1705-7.

50. Vivanti G, Paynter J, Duncan E, et al. Effectiveness and feasibility of the Early Start Denver Model implemented in a group-based community childcare setting. J Autism Dev Disord. 2014;44(12):314053.

51. Patel P, Kieling C, Maulik PK, Divan G. Improving access to care for children with mental disorders: A global perspective. Arch Dis Child. 2013;98:323-7.

52. Divan G, Hamdani S, Vajartkar V, et al. Adapting an evidence-based intervention for autism spectrum disorder for scaling up in resource-constrained settings: the development of the PASS intervention in South Asia. Glob Health Action. 2015;8(1):27278. doi: 10.3402/gha. v8.27278.

53. World Health Organization. Task shifting: rational redistribution of tasks among health workforce teams: global recommendations and guidelines. Geneva: World Health Organization. 2008.

54. Ministerio de Salud. Guía de Práctica Clínica de Detección y Diagnóstico Oportuno de los trastornos del Espectro Autista (TEA). Santiago, 2011. Disponible en: http://www.minsal.cl/portal/url/item/ bd81e3a09ab6c3cee040010164012ac2.pdf. última visita 10-06-2019. 